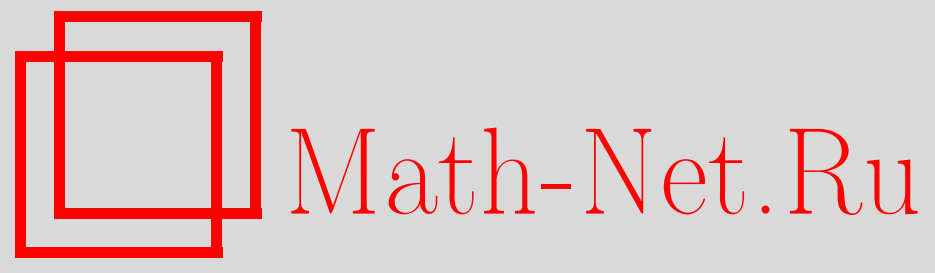

О. И. Череватенко, Многообразия линейных алгебр полиномиального роста, Вестн. Сам. гос. техн. ун-та. Сер. Физ.-мат. науки, 2013, выпуск 4(), 7-14

DOI: https://doi.org/10.14498/vsgtu1262

Использование Общероссийского математического портала MathNet.Ru подразумевает, что вы прочитали и согласны с пользовательским соглашением

http: //www.mathnet.ru/rus/agreement

Параметры загрузки:

IP: 34.229 .45 .116

26 апреля 2023 г., 12:51:34

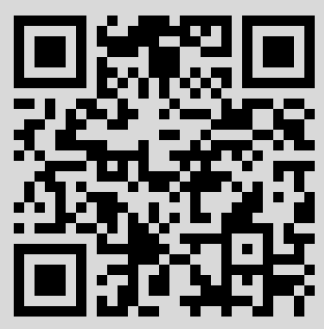




\title{
Алгебра
}

\author{
УДК 512.572
}

\section{МНОГООБРАЗИЯ ЛИНЕЙНЫХ АЛГЕБР ПОЛИНОМИАЛЬНОГО РОСТА}

\section{О. И. Череватенко}

Ульяновский государственный педагогический университет имени И. Н. Ульянова, Россия, 432063, Ульяновск, пл. 100-летия со дня рождения В. И. Ленина, 4.

E-mail: chai@pisem.net

Приведён обзор результатов о многообразиях линейных алгебр полиномиалъного роста. Приводятся эквивалентные условия полиномиальности роста многообразий ассоциативных алгебр, многообразий алгебр Ли, многообразий алгебр Лейбница, многообразий алгебр Пуассона и многообразий алгебр Лейбница-Пуассона. Показано, что при изучении многообразий линейных алгебр полиномиалъного роста важную роль играют многообразия почти полиномиалъного роста.

Ключевые слова: ассочиативная алгебра, алгебра Пуассона, алгебра Ли, многообразие алгебр, рост многообразия.

Введение. На протяжении всей работы предполагается, если это специально не оговорено, что основное поле имеет нулевую характеристику.

Пусть $K(X)$ - свободная алгебра над полем $K$, где $X=\left\{x_{1}, x_{2}, \ldots\right\}-$ счётное множество свободных образующих. Обозначим через $A$ некоторую $P I$-алгебру. Совокупность всех тождеств алгебры $A$ образует $T$-идеал $I d(A)$ в свободной алгебре $K(X)$. Пусть $\mathbf{V}$ - многообразие алгебр, порождённое алгеброй $A$. Тогда алгебра $K(X, \mathbf{V})=K(X) / I d(A)$ является относительно свободной алгеброй многообразия $\mathbf{V}$.

Пусть $P_{n}$ - подпространство в пространстве $K(X)$, состоящее из всех полилинейных элементов степени $n$ от переменных $x_{1}, x_{2}, \ldots, x_{n}$. В случае основного поля нулевой характеристики вся информация о многообразии $\mathbf{V}$ содержится в его полилинейных компонентах $P_{n}(\mathbf{V})=P_{n} /\left(P_{n} \cap \operatorname{Id}(\mathbf{V})\right), n=$ $=1,2, \ldots$ Асимптотическое поведение последовательности $c_{n}(\mathbf{V})=\operatorname{dim} P_{n}(\mathbf{V})$, $n=1,2, \ldots$, называют ростом многообразия $\mathbf{V}$. Говорят, что многообразие $\mathbf{V}$ имеет полиномиальный рост, если существуют такие константы $C$ и $k$, что для любого $n$ выполнено неравенство $c_{n}(\mathbf{V}) \leqslant C n^{k}$.

Пространство $P_{n}(\mathbf{V})$ наделено структурой левого $S_{n}$-модуля, где $S_{n}-$ симметрическая группа степени $n$. Напомним, что последовательность $\lambda=$ $=\left(\lambda_{1}, \lambda_{2}, \ldots, \lambda_{k}\right)$ называют разбиением числа $n$ и обозначают $\lambda \vdash n$, если $\lambda_{1}+\lambda_{2}+\cdots+\lambda_{k}=n$ и $\lambda_{1} \geqslant \lambda_{2} \geqslant \ldots \geqslant \lambda_{k}>0$. Пусть $\chi_{\lambda}$ - характер неприводимого представления симметрической группы, соответствующий разбиению $\lambda$ числа $n$. Тогда в силу вполне приводимости модуля $P_{n}(\mathbf{V})$ для многообразия $\mathbf{V}$ имеет место разложение

$$
\chi_{n}(\mathbf{V})=\chi_{n}\left(P_{n}(\mathbf{V})\right)=\sum_{\lambda \vdash n} m_{\lambda}(\mathbf{V}) \chi_{\lambda},
$$

Ольга Ивановна Череватенко (к.ф.-м.н.), доцент, каф. высшей математики. 
где $m_{\lambda}(\mathbf{V})$ - степени неприводимых представлений, соответствующих разбиению $\lambda$ числа $n$. Кодлина многообразия определяется как сумма

$$
l_{n}(\mathbf{V})=\sum_{\lambda \vdash n} m_{\lambda}(\mathbf{V})
$$

Если $\lambda$-разбиение некоторого числа $n$, то будем обозначать символом $\lambda^{\prime}$ сопряженное разбиение к $\lambda$.

В следующей теореме приводится критерий полиномиального роста для общего случая.

Теорема 1 [1]. Пусть V - некоторое многообразие линейных алгебр. Последовательность $\left\{c_{n}(\mathbf{V})\right\}_{n \geqslant 1}$ ограничена полиномом тогда и только тогда, когда выполнены следующие условия:

(i) существует такая константа $C$, что в сумме $(1) m_{\lambda}(\mathbf{V})=0$ в случае, если либо выполнено условие $n-\lambda_{1}>C$, либо $n-\lambda_{1}^{\prime}>C$;

(ii) существуют такие константы $C$ и $k$, что для любого $n$ существует $m \leqslant C n^{k}$ расстановок скобок $T_{1}, T_{2}, \ldots, T_{m}$ таких, что любой элемент $f \in P_{n}(\mathbf{V})$ может быть записан как

$$
f=\sum_{i=1}^{m} f_{i} \quad(\bmod \operatorname{Id}(\mathbf{V}))
$$

где $f_{i} \in P_{n}^{T_{i}}(\mathbf{V})$.

Далее будут приведены более конкретные эквивалентные условия полиномиального роста для конкретных случаев.

Договоримся опускать скобки при их левонормированной расстановке:

$$
((a b) c)=a b c
$$

Ассоциативные алгебры. В теории ассоциативных алгебр очень важную роль играет бесконечно порожденная алгебра Грассмана $\Lambda$ и алгебра верхнетреугольных матриц порядка 2 , которую обозначим через $U T_{2}$.

Теорема 2 [2]. Для алгебры $\Lambda$ верны следуюшие утверждения:

(i) полилинейное тождество $[x, y, z]=0$ порождает идеал тождеств алгебры Грассмана $\Lambda$;

(ii) рост многообразия $\operatorname{var}(\Lambda)$, порождённого алгеброй $\Lambda$, является почти полиномиальным, причём $c_{n}(\Lambda)=2^{n-1}$

(iii) базис полилинейной компоненты $P_{n}(\Lambda)$ состоит из элементов вида

$$
\left[x_{i_{1}}, x_{i_{2}}\right] \ldots\left[x_{i_{2 p-1}}, x_{i_{2 p}}\right] x_{j_{1}} x_{j_{2}} \ldots x_{j_{q}}
$$

где $\left\{i_{1}, \ldots, i_{2 p}, j_{1}, \ldots, j_{q}\right\}=\{1, \ldots, n\}, i_{1}<i_{2}<\cdots<i_{2 p}, j_{1}<\cdots<j_{q}$.

Теорема 3 [2]. Для алгебры UT 2 верны следующие утверждения:

(i) полилинейное тождество $\left[x_{1}, x_{2}\right]\left[x_{3}, x_{4}\right]=0$ порождает идеал тождеств алгебры $U T_{2}$

(ii) рост многообразия var $\left(U T_{2}\right)$, порождённого алгеброй $U T_{2}$, является почти полиномиальным, причём $c_{n}\left(U T_{2}\right)=2^{n-1}(n-2)+2$; 
(iii) базис полилинейной компоненты $P_{n}\left(U T_{2}\right)$ состоит из элементов вида

$$
\left[x_{i_{1}}, x_{i_{2}}, \ldots, x_{i_{p}}\right] x_{j_{1}} x_{j_{2}} \ldots x_{j_{q}}
$$

где $\left\{i_{1}, \ldots, i_{p}, j_{1}, \ldots, j_{q}\right\}=\{1, \ldots, n\}, j_{1}<\cdots<j_{q}$ и переменные в коммутаторе $\left[x_{i_{1}}, \ldots, x_{i_{p}}\right]$ упорядочены следующим образом:

$$
i_{1}>i_{2}<i_{3}<\cdots<i_{p}
$$

Теорема 4 [3]. Для многообразия ассоциативных алгебр $\mathbf{V}$ следующие условия эквивалентны:

(i) последовательность $\left\{c_{n}(\mathbf{V})\right\}_{n \geqslant 1}$ ограничена полиномом;

(ii) $U T_{2} \notin \mathbf{V}, \Lambda \notin \mathbf{V}$;

(iii) существует такая константа $C$, что в сумме $(1) m_{\lambda}(\mathbf{V})=0$ в случае, если выполнено условие $n-\lambda_{1}>C$;

(iv) кодлина многообразия $\mathbf{V}$ является конечной.

Алгебры Ли. Пусть $\mathbf{N}_{s} \mathbf{A}$ - многообразие алгебр Ли, определяемое тождеством $\left[\left[x_{1}, x_{2}\right], \ldots,\left[x_{2 s+1}, x_{2 s+2}\right]\right]=0$. В работе [4] показано, что многообразие $\mathbf{N}_{2} \mathbf{A}$ имеет почти полиномиальный рост.

Теорема $5[5,6]$. Для многообразия алгебр Ли $\mathbf{V}$ следующие условия эквивалентны:

(i) многообразие $\mathbf{V}$ имеет полиномиалъный рост;

(ii) для некоторого $s$ выполнено условие $\mathbf{N}_{2} \mathbf{A} \nsubseteq \mathbf{V} \subset \mathbf{N}_{s} \mathbf{A}$;

(iii) существует такая константа $C$, что в сумме $(1) m_{\lambda}(\mathbf{V})=0$ в случае, если выполнено условие $n-\lambda_{1}>C$.

Обозначим через $\mathbf{A}^{2}$ метабелево многообразие алгебр Ли, которое определяется тождеством $\left[\left[x_{1}, x_{2}\right],\left[x_{3}, x_{4}\right]\right]=0$. Хорошо известно, что многообразие $\mathbf{A}^{2}$ является наименьшим многообразием среди всех многообразий алгебр Ли роста не ниже полиномиального, то есть если рост некоторого многообразия $\mathbf{V}$ не ниже полиномиального, то $\mathbf{A}^{2} \subseteq \mathbf{V}$.

Алгебры Лейбница. Алгебры Лейбница - неантикоммутативные алгебры Ли, которые определяются тождеством Лейбница

$$
[[x, y], z]=[[x, z], y]+[x,[y, z]]
$$

Тождество Лейбница представляет собой следующее условие: оператор правого умножения на любой элемент алгебры является дифференцированием.

Обозначим через $\widehat{\mathbf{N}_{s} \mathbf{A}}$ многообразие алгебр Лейбница, определяемое тождеством

$$
\left[\left[x_{1}, x_{2}\right], \ldots,\left[x_{2 s+1}, x_{2 s+2}\right]\right]=0 .
$$

Понятно, что многообразие $\widetilde{\mathbf{N}_{s} \mathbf{A}}$ с тождеством $[x, x]=0-$ в точности многообразие $\mathbf{N}_{s} \mathbf{A}$.

Обозначим через $\widetilde{\mathbf{V}}_{1}$ многообразие алгебр Лейбница, определенное тождеством $\left[x_{1},\left[x_{2}, x_{3}\right],\left[x_{4}, x_{5}\right]\right]=0$. Данное многообразие имеет почти полиномиальный рост (см. [7]).

Теорема $6[8,9]$. Для многообразия алгебр Лейбнииа $\mathbf{V}$ следующие условия эквивалентнь: 
(i) многообразие $\mathbf{V}$ имеет полиномиальный рост;

(ii) для некоторого $s$ выполнено условие

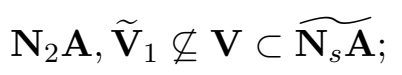

(iii) существует такая константа $C$, что в сумме $(1) m_{\lambda}(\mathbf{V})=0$ в случае, если выполнено условие $n-\lambda_{1}>C$.

Пусть В - многообразие алгебр Лейбница, определённое тождеством

$$
[x,[y, z]]=0 .
$$

В работе [10] показано, что многообразия $\mathbf{A}^{2}$ и $\mathbf{B}$ исчерпывают весь список минимальных многообразий среди всех многообразий алгебр Лейбница роста не ниже полиномиального, то есть если рост некоторого многообразия $\mathbf{V}$ не ниже полиномиального, то либо $\mathbf{A}^{2} \subseteq \mathbf{V}$, либо $\mathbf{B} \subseteq \mathbf{V}$.

Алгебры Пуассона. Алгебра $A=A(+, \cdot,\{\}, K$,$) над произвольным$ полем $K$ называется алгеброй Пуассона, если $A(+, \cdot, K)$ - ассоциативная коммутативная алгебра с единицей, $A(+,\{\}, K$,$) - алгебра Ли с операци-$ ей умножения $\{$,$\} , которая называется скобкой Пуассона, и выполняется$ правило Лейбница:

$$
\{a \cdot b, c\}=a \cdot\{b, c\}+\{a, c\} \cdot b, \quad a, b, c \in A .
$$

Пусть $\Lambda$ - бесконечномерная алгебра Грассмана с единицей и операцией умножения $\wedge$. Введём в алгебре $\Lambda$ два новых умножения:

$$
a \cdot b=\frac{1}{2}(a \wedge b+b \wedge a), \quad\{a, b\}=a \wedge b-b \wedge a, \quad a, b \in \Lambda .
$$

Нетрудно проверить, что алгебра $(\Lambda,+, \cdot,\{\}$,$) будет алгеброй Пуассона, ко-$ торую обозначим через $G$.

Теорема 7 [11]. Для алгебры $G$ верны следующие утверждения:

(i) полилинейное тождество $\{x, y, z\}=0$ порождает идеал тождеств алгебры Пуассона $G$;

(ii) рост многообразия $\operatorname{var}(G)$, порождённого алгеброй $G$, является почти полиномиальным, причём $c_{n}(G)=2^{n-1}$;

(iii) базис полилинейной компоненты $P_{n}(G)$ состоит из элементов вида

$$
\left\{x_{i_{1}}, x_{i_{2}}\right\} \cdot \ldots \cdot\left\{x_{i_{2 p-1}}, x_{i_{2 p}}\right\} \cdot x_{j_{1}} \cdot x_{j_{2}} \cdot \ldots \cdot x_{j_{q}},
$$

әде $\left\{i_{1}, \ldots, i_{2 p}, j_{1}, \ldots, j_{q}\right\}=\{1, \ldots, n\}, i_{1}<i_{2}<\cdots<i_{2 p}, j_{1}<\cdots<j_{q}$.

Пусть $A_{L}$ - некоторая ненулевая алгебра Ли с лиевым умножением [, ] над произвольным полем $K$. Рассмотрим векторное пространство $A=A_{L} \oplus K$, в котором определим операции · и $\{$,$\} следующим образом:$

$$
\begin{gathered}
(a+\alpha) \cdot(b+\beta)=(\beta a+\alpha b)+\alpha \beta, \\
\{a+\alpha, b+\beta\}=[a, b], \quad a, b \in A_{L}, \quad \alpha, \beta \in K .
\end{gathered}
$$

Тогда полученная алгебра $(A,+, \cdot,\{\}, K$,$) будет являться алгеброй Пуас-$ сона. 
Пусть $U_{2}^{L}$ - двумерная метабелева алгебра Ли с базисом $\{a, b\}$ и таблицей умножения $[a, b]=a,[b, a]=-a,[a, a]=[b, b]=0$. Обозначим через $U_{2}$ алгебру Пуассона $U_{2}^{L} \oplus K$ с операциями (2).

Теорема 8 [12]. Для алгебры $U_{2}$ верны следующие утверждения:

(i) полилинейные тождества

$$
\left\{x_{1}, x_{2}\right\} \cdot\left\{x_{3}, x_{4}\right\}=0, \quad\left\{\left\{x_{1}, x_{2}\right\},\left\{x_{3}, x_{4}\right\}\right\}=0
$$

порождают идеал тождеств алгебры Пуассона $U_{2}$;

(ii) рост многообразия $\operatorname{var}\left(U_{2}\right)$, порождённого алгеброй $U_{2}$, является почти полиномиальным, причём $c_{n}\left(U_{2}\right)=2^{n-1}(n-2)+2$;

(iii) базис полилинейной компоненты $P_{n}\left(U_{2}\right)$ состоит из элементов вида

$$
\left\{x_{i_{1}}, x_{i_{2}}, \ldots, x_{i_{p}}\right\} \cdot x_{j_{1}} \cdot x_{j_{2}} \cdot \ldots \cdot x_{j_{q}}
$$

где $\left\{i_{1}, \ldots, i_{p}, j_{1}, \ldots, j_{q}\right\}=\{1, \ldots, n\}, j_{1}<\cdots<j_{q}$ и переменные в мономе $\left\{x_{i_{1}}, \ldots, x_{i_{p}}\right\}$ упорядочены следующим образом:

$$
i_{1}>i_{2}<i_{3}<\cdots<i_{p}
$$

Следующая теорема показывает, что в случае алгебр Пуассона алгебры $U_{2}$ и $G$ играют ту же роль, что и алгебры $U T_{2}$ и $\Lambda$ в ассоциативном случае.

Теорема 9 [12]. Для многообразия алгебр Пуассона $\mathbf{V}$ следующие условия эквивалентны:

(i) последовательность $\left\{c_{n}(\mathbf{V})\right\}_{n \geqslant 1}$ ограничена полиномом;

(ii) $U_{2} \notin \mathbf{V}, G \notin \mathbf{V}$;

(iii) существует такая константа $C$, что в сумме $(1) m_{\lambda}(\mathbf{V})=0$ в случае, если выполнено условие $n-\lambda_{1}>C$;

(iv) кодлина многообразия $\mathbf{V}$ является конечной.

Пусть $N_{3}^{L}$ - трёхмерная нильпотентная алгебра Ли с базисом $\{a, b, c\}$ и таблицей умножения $[b, a]=c,[a, c]=[b, c]=0$. Обозначим через $N_{3}$ алгебру Пуассона $N_{3}^{L} \oplus K$ с операциями (2).

Пусть $\Lambda_{2 n}$ - алгебра Грассмана с единицей и $2 n$ образующими элементами $\left\{e_{1}, \ldots, e_{2 n}\right\}$. Определим в алгебре $\Lambda_{2 n}$ операции умножения (). Полученную алгебру Пуассона обозначим через $G_{2 n}$.

Следующая теорема показывает, что многообразие, порождённое либо алгеброй $N_{3}$, либо алгеброй $G_{2}$, является наименьшим многообразием алгебр Пуассона в классе всех многообразий алгебр Пуассона, имеющих рост не ниже полиномиального.

Теорема 10 [13]. Для алгебр $N_{3}$ и $G_{2}$ верны следующие утвержсдения:

(i) $\operatorname{var}\left(N_{3}\right)=\operatorname{var}\left(G_{2}\right)=\mathbf{V}$;

(ii) тождества $\left\{x_{1}, x_{2}, x_{3}\right\}=0,\left\{x_{1}, x_{2}\right\} \cdot\left\{x_{3}, x_{4}\right\}=0$ порождают идеал тождеств многообразия $\mathbf{V}$;

(iii) для любого $n$ выполнено равенство $c_{n}(\mathbf{V})=1+n(n-1) / 2$;

(iv) многообразие $\mathbf{V}$ является наименъшим многообразием среди всех многообразий алгебр Пуассона роста не ниже полиномиального, то есть если рост некоторого многообразия $\mathbf{W}$ не ниже полиномиального, то $\mathbf{V} \subseteq \mathbf{W}$. 
Алгебры Лейбница-Пуассона. В данном пункте рассматриваются алгебры Пуассона с неантикоммутативной операцией $\{$,$\} , которые будем назы-$ вать алгебрами Лейбница-Пуассона. Более точно векторное пространство $A$ над полем $K$ с двумя $K$-биллинейными операциями умножения $\cdot$ и $\{$, $\}$ называется алгеброй Лейбница-Пуассона, если относительно операции · пространство $A$ является коммутативной ассоциативной алгеброй с единицей, относительно операции $\{$,$\} - алгеброй Лейбница, и данные операции$ связаны правилами

$$
\{a \cdot b, c\}=a \cdot\{b, c\}+\{a, c\} \cdot b, \quad\{c, a \cdot b\}=a \cdot\{c, b\}+\{c, a\} \cdot b,
$$

где $a, b, c \in A$.

Обозначим через $\Gamma_{n}$ пространство в свободной алгебре Лейбница-Пуассона, состоящее из всех полилинейных элементов степени $n$ от переменных $x_{1}, \ldots, x_{n}$ и являющееся линейной оболочкой элементов вида

$$
\left\{x_{i_{1}}, \ldots, x_{i_{s}}\right\} \cdot \ldots \cdot\left\{x_{j_{1}}, \ldots, x_{j_{t}}\right\}, \quad s \geqslant 2, \ldots, t \geqslant 2
$$

Теорема 11 [14]. Пусть V - нетривиалъное многообразие алгебр Лейбнииа-Пуассона над произвольным полем. Тогда либо

(i) $c_{n}(\mathbf{V}) \geqslant 2^{n-1}$ для любого $n$, либо

(ii) найдётся такой многочлен $f(x) \in \mathbb{Q}[x]$, что для всех достаточно больших $n$ будет выполнено равенство $c_{n}(\mathbf{V})=f(n)$.

Пусть V - некоторое многообразие алгебр Лейбница-Пуассона, $\operatorname{Id}(\mathbf{V})$ идеал тождеств многообразия V. Обозначим

$$
\Gamma_{n}(\mathbf{V})=\Gamma_{n} /\left(\Gamma_{n} \cap I d(\mathbf{V})\right), \quad \gamma_{n}(\mathbf{V})=\operatorname{dim} \Gamma_{n}(\mathbf{V})
$$

Теорема 12 [14]. Для многообразия алгебр Лейбница-Пуассона V над произвольным полем следующие условия эквивалентны:

(i) последовательность $\left\{c_{n}(\mathbf{V})\right\}_{n \geqslant 1}$ ограничена полиномом;

(ii) для некоторого $m \geqslant 2$ в $\mathbf{V}$ выполнены полилинейные тождества

$$
\left\{x_{1}, \ldots, x_{m}\right\}=0, \quad\left\{x_{1}, y_{1}\right\} \cdot \ldots \cdot\left\{x_{m}, y_{m}\right\}=0
$$

(iii) найдётся такое число $N$, что для любого $n>N$ выполнено равенство $\gamma_{n}(\mathbf{V})=0$

(iv) найдётся такое число $N$, что для любого $n \geqslant N$ будет выполнено равенство

$$
c_{n}(\mathbf{V})=1+\sum_{k=2}^{N} C_{n}^{k} \cdot \gamma_{k}(\mathbf{V})
$$

Рассмотрим двумерную алгебру Лейбница $L_{2}$ над полем $K$ с базисом $a, b$ и таблицей умножения $[a, b]=a,[a, a]=[b, b]=[b, a]=0$. Обозначим через $B^{2}$ алгебру Лейбница-Пуассона $L_{2} \oplus K$ с операциями (2).

Теорема 13 [14]. Для алгебры Лейбнииа-Пуассона $B^{2}$ верны следующие утверждения: 
(i) полилинейные тождества

$$
\left\{x_{1}, x_{2}\right\} \cdot\left\{x_{3}, x_{4}\right\}=0, \quad\left\{x_{1},\left\{x_{2}, x_{3}\right\}\right\}=0
$$

порождают идеал тождеств алгебры $B^{2}$;

(ii) рост многообразия $\operatorname{var}\left(B^{2}\right)$, порождённого алгеброй $B^{2}$, является почти полиномиальным, причём для любого натурального $n$ выполнено равенство

$$
c_{n}\left(B^{2}\right)=n \cdot 2^{n-1}-n+1 ;
$$

(iii) базис полилинейной компоненты $P_{n}\left(B^{2}\right)$ состоит из элементов вида

$$
x_{i_{1}} \cdot \ldots \cdot x_{i_{s}} \cdot\left\{x_{j_{1}}, \ldots, x_{j_{t}}\right\},
$$

где $\left\{i_{1}, \ldots, i_{s}, j_{1}, \ldots, j_{t}\right\}=\{1, \ldots, n\}, i_{1}<\cdots<i_{s}, j_{2}<\cdots<j_{t}$.

Обозначим через $\mathbf{W}_{s}$ многообразие алгебр Лейбница-Пуассона, порождённое полилинейным тождеством

$$
\left\{x_{1}, y_{1}\right\} \cdot \ldots \cdot\left\{x_{s}, y_{s}\right\}=0 \text {. }
$$

Теорема 14 [15]. Для многообразия алгебр Лейбнииа-Пуассона $\mathbf{V}$ следующие условия эквивалентны:

(i) последовательность $\left\{c_{n}(\mathbf{V})\right\}_{n \geqslant 1}$ ограничена полиномом;

(ii) $U_{2} \notin \mathbf{V}, B^{2} \notin \mathbf{V}$ и для некоторого $s \geqslant 2$ выполнено включение $\mathbf{V} \subset \mathbf{W}_{s}$;

(iii) существует такая константа $C$, что в сумме $(1) m_{\lambda}(\mathbf{V})=0$ в случае, если выполнено условие $n-\lambda_{1}>C$.

\section{БИБЛИОГРАФИЧЕСКИЙ СПИСОК}

1. A. Giambruno, S. P. Mishchenko, "Polynomial growth of the codimentions: a characterization" // Proc. Amer. Math. Soc., 2010. Vol. 138, no. 3. Pp. 853-859.

2. V. Drensky, Free algebras and PI-algebras. Graduate course in algebra. Singapore: SpringerVerlag, 2000. xii+271 pp.

3. А. Р. Кемер, "Шпехтовость Т-идеалов со степенным ростом коразмерностей" // Сиб. матем. журнал, 1978. Т. 19, № 1. С. 54-69. [A. R. Kemer, "Spechtianess of T-ideals with polynomial growth of the co-dimensions" // Sib. Mat. Zh., 1978. Vol.19, no. 1. Pp. 54-69].

4. С. П. Мищенко, "Многообразия алгебр Ли с двуступенно нильпотентным коммутантом" // Весиі Акадэміi навук БССР. Сер. фiз.-мат. навук, 1987. №6. С. 39-43. [ "Varieties of Lie algebras with two-step nilpotent commutant" // Vestsi Akad. Navuk BSSR. Ser. Fiz.-Mat. Navuk, 1987. no. 6. Pp. 39-43].

5. И. И. Бенедиктович, А. Е. Залесский, "Т-идеалы свободных алгебр Ли с полиномиальным ростом последовательности коразмерностей” // Весиі Акадэміi навук БССР. Сер. giз.-мam. навук., 1980. № 3. C. 5-10. [I. I. Benediktovich, A. E. Zalesskiy, "T-ideals of free Lie algebras with polynomial growth of a sequence of codimensionalities" // Vestsi Akad. Navuk BSSR Ser. Fiz.-Mat. Navuk, 1980. no. 3. Pp. 5-10].

6. C. П. Мищенко, "О многообразиях полиномиального роста алгебр Ли над полем характеристики нуль"// Матем. заметки, 1986. Т. 40, №6. С. 713-721; англ. пер.: S. P. Mishchenko, "Varieties of polynomial growth of Lie algebras over a field of characteristic zero" // Math. Notes, 1986. Vol.40, no. 6. Pp. 901-905.

7. S. Mishchenko, A. Valenti, "A Leibniz variety with almost polynomial growth" // J. Pure Appl. Algebra, 2005. T. 202, № 1-3. C. 82-101. 
8. С. П. Мищенко, О. И. Череватенко, "Необходимые и достаточные условия полиномиальности роста многообразия алгебр Лейбница" // Фундамент. и прикл. матем., 2006. T. 12, № 8. C. 207-215; англ. пер.: S. P. Mishchenko, O. I. Cherevatenko, "Necessary and sufficient conditions for a variety of Leibniz algebras to have polynomial growth" // J. Math. Sci., 2008. Vol. 152, no. 2. Pp. 282-287.

9. С. П. Мищенко, О. И. Череватенко, "Многообразия алгебр Лейбница слабого роста" // Вестн. СамГУ. Естественнонаучн. сер., 2012. №9(49). С. 19-23. [S. P. Mishchenko, O. I. Cherevatenko, "Variety of Leibniz algebras of weak growth" // Vestnik SamGU. Estestvenno-Nauchnaya Ser., 2012. no. 9(49). Pp. 19-23].

10. О. И. Череватенко, "О нильпотентных алгебрах Лейбница" // Научные ведомости БелГУ. Математика. Физика, 2012. №17(136). С. 132-136. [O. I. Cherevatenko, "On nilpotent Leibnitz algebras" // Nauchnyye vedomosti BelGU. Matematika. Fizika, 2012. no. 17(136). Pp. 132-136].

11. S. P. Mishchenko, V. M. Petrogradsky, A. Regev, "Poisson PI algebras"// Trans. Amer. Math. Soc., 2007. T. 359, № 10. C. 4669-4694.

12. С. М. Ращеев, "Эквивалентные условия полиномиальности роста многообразий алгебр Пуассона" // Вестн. Моск. унив. Сер. 1. Математика. Механика, 2012. Т. 67, № 5. С. 813; англ. пер.:S. M. Ratseev, "Equivalent conditions of polynomial growth of a variety of Poisson algebras" // Mosc. Univ. Math. Bull., 2012. Vol.67, no. 5-6. Pp. 195-199.

13. С. М. Ращеев, "Алгебры Пуассона полиномиального роста"// Сиб. матем. журн., 2013. Т. 54, № 3. С. 700-711; англ. пер.: S. M. Ratseev, "Poisson algebras of polynomial growth" // Siberian Math. J., 2013. Vol.54, no. 3. Pp. 555-565.

14. C. M. Рацеев, "Коммутативные алгебры Лейбница-Пуассона полиномиального роста" // Вестн. СамГУ. Естественнонаучн. сер., 2012. №3/1(94). С. 54-65. [S. M. Ratseev, "Commutative Leibniz-Poisson algebras of polynomial growth" // Vestnik SamGU. Estestvenno-Nauchnaya Ser., 2012. no. 3/1(94). Pp. 54-65].

15. С. М. Рацеев, "Необходимые и достаточные условия полиномиальности роста многообразий алгебр Лейбница-Пуассона" // Изв. вузов. Матем., 2014. № 3. С. 33-39; англ. пер.: S. M. Ratseev, "Necessary and sufficient conditions of polynom ial growth of varieties of Leibniz-Poisson algebras" // Russian Math. (Iz. VUZ), 2014. Vol. 58, no. 3 (to appear).

Поступила в редакцию 27/IX/2013;

в окончательном варианте $-12 / \mathrm{X} / 2013$.

MSC: 16R10, 17A32, 17B01, 17B63

\section{VARIETIES OF LINEAR ALGEBRAS OF POLYNOMIAL GROWTH}

\section{O. I. Cherevatenko}

Ulyanovsk State I. N. Ulyanov Pedagogical University,

4, Ploshchad' 100-letiya so dnya rozhdeniya V. I. Lenina, 432063, Ulyanovsk, Russia.

E-mail: chai@pisem.net

The paper is survey of results of investigations on varieties of linear algebras of polynomial growth. We give equivalent conditions of the polynomial codimension growth of a variety of associative algebras, Lie algebras, Leibniz algebras, Poisson algebras, Leibniz-Poisson algebras. It is shown that in the study of varieties of linear algebras of polynomial growth varieties of almost polynomial growth play an important role.

Keywords: associative algebra, Poisson algebra, Lie algebra, variety of algebras, growth of a variety.

Original article submitted 27/IX/2013; revision submitted 12/X/2013.

Olga I. Cherevatenko (Ph. D. Phys. \& Math.), Associate Professor, Dept. of Higher Mathematics. 